\title{
The Effects of Severe Iron Deficiency Anemia on T-wave Intervals
}

\author{
Huseyin Avni Findikli ${ }^{*}$, Ayse Sahin Tutak ${ }^{2}$ \\ ${ }^{1}$ Department of Internal Medicine, Kahramanmaras Necip Fazil City Hospital, Turkey \\ ${ }^{2}$ Department of Internal Medicine, Adiyaman University, Turkey
}

*Corresponding Author: Huseyin Avni Findikli, Department of Internal Medicine, Kahramanmaraş Necip Fazil City Hospital, Turkey, USA; E-mail: dr-avni@hotmail.com; Tel: +90-3442282800

Received: 06 December 2018; Accepted: 14 December 2018; Published: 17 December 2018

\begin{abstract}
Aim: Changes in the iron level in the myocardium may be affected by sodium and potassium channels in myocytes and there may be a contribution to the etiology of cardiac arrhythmia. The aim of this study was to examine for the first time in literature how the Tp-e interval, which has been accepted in recent years as a strong ventricular repolarisation index, is affected in female patients with severe iron deficiency anemia.
\end{abstract}

Material and Methods: The study included a total of 64 participants, as a study group of 34 patients diagnosed with severe iron deficiency anemia $\left(\mathrm{Hgb}<8 \mathrm{~g} / \mathrm{dL}\right.$ Ferritin $\left.<14 \mathrm{mcg} / \mathrm{L}, \mathrm{MCV}<80 \mathrm{~mm}^{3}\right)$ and a control group of 34 subjects. To determine the QTc and Tp-Te interval durations, manual examination was made of manual precordial V5 lead ECGs taken routinely on the presentation of all participants.

Results: The Tp-e interval was determined to be statistically significantly shorter in the patients $(78.97 \pm 8.63 \mathrm{~ms})$ compared to the control group $(84.57 \pm 10.13 \mathrm{~ms})(\mathrm{p}<0.05)$. A positive relationship was determined between Tp-e and QTc durations and $\mathrm{Hgb}$ and ferritin levels $(\mathrm{p}<0.05)$.

Conclusion: The results of the study showed that the QTc duration and the Tp-e duration were shorter in female patients with iron deficiency anemia, and therefore iron deficiency anemia can be discounted as a cause of prolonged QTc and Tp-e durations.

Keywords: Anemia; Iron deficiency; t-peak; t-end interval

\section{Introduction}

Iron deficiency anemia is the most common cause of anemia worldwide. More than a quarter of the global population has anemia and half of these cases are formed of iron deficiency anemia [1, 2]. Correspondingly, in developed countries, Archives of Clinical and Biomedical Research- http://archclinbiomedres.com/ - Vol. 2 No. 6 - Dec 2018. [ISSN 2572-5017] 
gynecological bleeding in women during the menstrual period and blood loss from the gastrointestinal system in males and postmenopausal females are the most important etiological factors [3]. Attention has been drawn to the relationship between anemia and cardiovascular disease. Anemia can increase the fluid in heart and heart rate, can be a cause of left ventricle hypertrophy, activation of the sympathetic nerve system and stimulation of the renin angiotensin aldosterone system, and is closely related to chronic inflammation and increased oxidative stress [4].

The shape of the T-wave is a reflection of the ventricular myocardial repolarisation and includes valuable information about this process. Therefore, the morphological characteristics of the T-wave have become a focus of increasing interest. The components of T-wave morphology have been shown to be relatively independent of pulse rate, in contrast to the QT interval. The Tp-e interval duration can be defined as a transmural dispersion index of repolarisation and in this context, it can be used as a predictive marker for the risk of developing life-threatening arrhythmia [5-6]. It is known that iron in the heart is suppressed in myocardial cells rather than from the interstitium, and previous studies have suggested that increased intracellular iron interacts with the cardiac electrical function by producing large numbers of free radicals or by impairing the selective function of $\mathrm{Na}+$ channels [7-9]. As an abnormal function of the $\mathrm{Na}+$ and $\mathrm{K}+$ channels may contribute to the etiology of cardiac arrhythmia such as prolonged QT syndrome, the aim of this study was to compare the TP-e and QT intervals in female patients with severe iron deficiency anemia and a control group.

\section{Subjects and Methods}

\subsection{Study population}

This cross-sectional, prospective study included 64 subjects as a patient group of 34 females and an age-matched control group of 30 females. The patient group were diagnosed with severe iron deficiency anemia determined from ferritin $(<14$ $\mathrm{mcg} / \mathrm{L}), \mathrm{MCV}\left(<80 \mathrm{~mm}^{3}\right)$, and $\mathrm{Hgb}(<8 \mathrm{~g} / \mathrm{dL})$ values lower than the reference intervals. The control group subjects were selected as healthy individuals not determined with iron deficiency anemia. In both groups, the voluntary participants had no chronic disease, and biochemistry laboratory results for electrolytes, fasting blood glucose, urea, creatinine, B12 and folic acid were within the reference intervals.

\subsection{Measurements}

Blood samples were taken after an 8-12-hour fast, and were centrifuged immediately. In the biochemical assays, measurements were taken using the photometric method with Abbott-Architect C16000 (Abbott, USA), the ion selective electrode method with Abbott-Architect C16000 (Abbott, USA), and the impedance and laser scanning method with CellDyne Ruby (Abbott, USA). Resting ECG (Nikon Kohden 1250 ECG machine) was recorded at $50 \mathrm{~mm} / \mathrm{sec}$ paper rate and was examined manually. The Tp-e interval was defined by the tangential method as the distance from the peak of the Twave to the end of the T-wave. The QT interval was defined as the time from the start of QRS to the reversal of the T-wave to the base line. The QTc interval was calculated using the Bazett formula. A precordial V5 probe was used for both measurements.

\section{Statistical Analysis}

Data obtained in the study were analyzed statistically using SPSS Statistics vn 21 software (SPSS Inc., Chicago, IL, USA). 
Conformity of the data to normal distribution was assessed with visual (histogram and probability graphs) and analytical methods (Kolmogorov-Smirnov test). Variance homogeneity was evaluated with the Levene test. In descriptive analyses, variables with normal distribution were stated as mean \pm standard deviation (SD) values. For the difference between groups of parametric variables, the Student's t-test was applied, and the correlation coefficients and statistical significance were calculated using the Pearson test. A total type1 error level of 5\% was accepted as the level of statistical significance in all the statistical evaluations.

\section{Results}

The mean age was similar in both study groups as $33.41 \pm 5.63$ years in the patient group and $33.13 \pm 3.64$ years in the control group ( $p>0.05$ ). The basal demographic, clinical and laboratory data of both groups are shown in Table 1 .

\begin{tabular}{|l|l|l|l|}
\hline Variables Total & $\begin{array}{l}\text { Controls } \\
\mathbf{( n = 3 0 )}\end{array}$ & $\begin{array}{l}\text { Patients } \\
\mathbf{( n = 3 4 )}\end{array}$ & $\boldsymbol{P}$ \\
\hline Age (years) & $33.13 \pm 3.64$ & $33.41 \pm 5.63$ & 0.818 \\
\hline BMI & $28.73 \pm 5.53$ & $29.44 \pm 2.79$ & 0.295 \\
\hline HR (bpm) & $71.60 \pm 4.08$ & $78.59 \pm 4.70$ & 0.005 \\
\hline SBP (mmHg) & $113.6 \pm 7,36$ & $111.8 \pm 9,67$ & 0.108 \\
\hline DBP (mmHg) & $73.93 \pm 4.08$ & $71.56 \pm 5.04$ & 0.044 \\
\hline Hgb (g/d) & $13.34 \pm 0,68$ & $6.48 \pm 1.32$ & $<.001$ \\
\hline Ferritin (ng/ml) & $20.22 \pm 3.53$ & $10.14 \pm 2,65$ & $<.001$ \\
\hline QTc Interval(ms) & $419.3 \pm 8.40$ & $412.9 \pm 11.34$ & 0.014 \\
\hline Tp-e Interval(ms) & $84.57 \pm 10.13$ & $78.97 \pm 8.63$ & 0.020 \\
\hline
\end{tabular}

Values are presented as mean \pm standard deviation, BMI-Body Mass Index; HR-Hearth Rate; SBP-Systolic Blood Pressure; DBP-Diastolic Blood Pressure; Hgb-Hemoglobin; QTc-Bezzet's correction; Tp-e-Tpeak and Tend interval.

Table 1: Demographic and biochemical characteristics of the controls and the patient groups.

Significant differences were determined between the groups in respect of Tp-e and QTc intervals. The Tp-e interval was measured as $78.97 \pm 8.63 \mathrm{~ms}$ in the patient group and $84.57 \pm 10.13 \mathrm{~ms}$ in the control group (Figure 1).

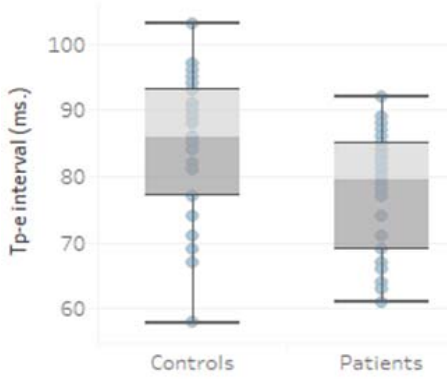

Figure 1: Comparison of Tp-e (Tpeak-Tend) interval between the study groups. 
The QTc interval was measured as $412.9 \pm 11.34$ in the patient group and $419.3 \pm 8.40$ in the control group. The Tp-e and QTc intervals were statistically significantly shorter in the patient group than in the control group ( $<<0.05$ ). In the correlation analyses, a positive correlation was determined between the Tp-e and QTc intervals and the Hgb and ferritin levels $(\mathrm{p}<0.05)$ (Table 2, Figure 2).

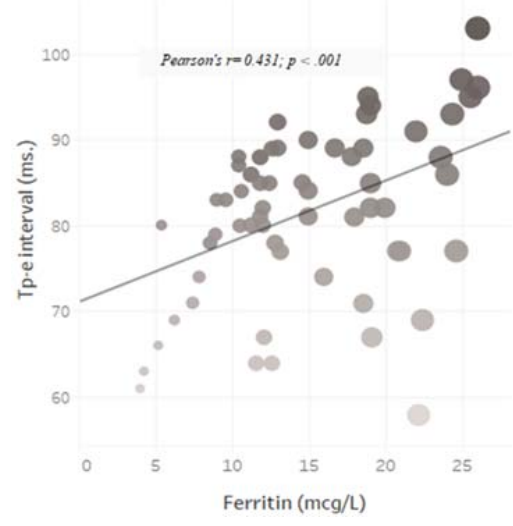

Figure 2: Correlation between $\mathrm{T}$ peak $\mathrm{T}$ end (Tp-e) interval time and Ferritin (Color shows Tp-e. Size shows Ferritin levels).

\begin{tabular}{|l|l|l|l|l|l|}
\hline Variables Tool & Hgb & Ferritin & QTc Interval & Tp-e Interval \\
\hline \multirow{2}{*}{ Hgb } & Pearson's r & - & & & \\
\cline { 2 - 6 } & p-value & - & & & \\
\hline \multirow{2}{*}{ Qerritin } & Pearson's r & 0.915 & - & & \\
\cline { 2 - 6 } & p-value & $<.001$ & - & & \\
\hline \multirow{2}{*}{ Tp-e Interval } & Pearson's r & 0.312 & 0.333 & - & \\
\cline { 2 - 6 } & p-value & 0.012 & 0.007 & - & - \\
\cline { 2 - 6 } & Pearson's r & 0.394 & 0.431 & 0.232 & - \\
\hline
\end{tabular}

Hgb-Hemoglobin; QTc-Bezzet's correction; Tp-e-Tpeak and Tend interval.

Table 2: Correlation of Tp-e and QTc with Hgb and Ferritin levels.

\section{Discussion}

To the best of our knowledge, this is the first study in literature to have examined how the Tp-e interval, which is a new and highly predictive arrhythmogenity index, is affected in patients with anemia. The results of the study showed that the measured Tp-e and QTc intervals were significantly shorter in the patients than in the control group. The Tp-e and QTc intervals were determined to be similarly affected in anemia patients and changes in both parameters were closely related to the severity of anemia.

Several studies have emphasized that the duration of the Tp-e interval could be affected depending on various clinical entities in individuals with no underlying heart disease and it could be a predictive parameter for potentially lifethreatening ventricular arrhythmia. To the best of our knowledge, there has been no previous study in the literature that has 
provided any data related to the effect of the Tp-e interval in patients with anemia and/or iron deficiency anemia. Previous studies have only encompassed the change in QTc interval in anemic patients. In a study of 30 non-pregnant females with severe iron deficiency anemia, Vitthal HK et al showed that the QTc interval was shorter in patients than in the control group and there was a significant positive correlation between serum ferritin level and the QTc interval [10]. In another study of non-pregnant females with severe iron deficiency anemia, positive correlations were also reported between serum ferritin or hemoglobin and QTc (12). The results of the current study were consistent with the literature in this respect.

Several electrocardiographic changes have been identified in patients with anemia, including ST segment depression, Twave inversion, extension or shortening of the QT interval, and reduced QRS complex amplitude [10,12, 13]. The increase in sympathetic excessive activity from the shortened QT interval with hypoxia and reduced myocardial oxygen source can be possibly held responsible for the pathophysiological connection between anemia and prolonged QT intervals [10, 13]. QT and Tp-e interval durations may be affected associated with autonomic tonus and this effect is less for the Tp-e duration. The reason for this can be said to be that $\mathrm{Tp}$-e evaluation, in contrast to conventional QT measurements, does not include the QRS complex and is therefore less affected by ventricular depolarization [14-16]. The results of the current study showed that shortened QTc and Tp-e were observed in the anemic group because of sympathetic excessive activity secondary to hyperdynamic circulation and the QTc duration was affected more.

There were some limitations to this study, primarily the small sample size and the cross-sectional design. No comparison was made of the ECGs of the same individuals in the anemic period and after treatment in respect of obtaining more homogenised Tp-Tp-e and QTc interval durations. However, a strong aspect of the study was the homogenous sample, as it was formed of females only.

In conclusion, the results of this study demonstrated how the Tp-e interval and QTc duration are affected in females with iron deficiency anemia, and as they were shortened, iron deficiency anemia can be excluded as a cause of prolonged QTc and Tp-e. There is a need for further large, prospective, randomized trials to determine the relationship between both regional and transmyocardial repolarization parameters and cardiac events.

\section{References}

1. Andrews NC. Iron metabolism: Iron deficiency and iron overload. Annual review of genomics and human genetics 1 (2000): 75-98.

2. Petry N, Olofin I, Hurrell RF, et al. The Proportion of Anemia Associated with Iron Deficiency in Low, Medium, and High Human Development Index Countries: A Systematic Analysis of National Surveys. Nutrients 8 (2016): 693.

3. Frewin R, Henson A, Provan D. ABC of clinical haematology. Iron deficiency anaemia. BMJ (Clinical research ed) 314 (1997): 360-363.

4. Zoppini G, Targher G, Chonchol M, et al. Anaemia, independent of chronic kidney disease, predicts all-cause and cardiovascular mortality in type 2 diabetic patients. Atherosclerosis 210 (2010): 575-580.

5. Baltazar RF. Basic and Bedside Electrocardiography. (1 ${ }^{\text {st }}$ Edn.). Lippincott Williams and Wilkins (2009). 
6. Yan GX, Antzelevitch C. Cellular basis for the normal T wave and the electrocardiographic manifestations of the long-QT syndrome. Circulation 98 (1998): 1928-1936.

7. Obejero-Paz CA, Yang T, Dong WQ, et al. Deferoxamine promotes survival and prevents electrocardiographic abnormalities in the gerbil model of iron-overload cardiomyopathy. The Journal of laboratory and clinical medicine 141 (2003): 121-130.

8. Rosenqvist M, Hultcrantz R. Prevalence of a haemochromatosis among men with clinically significant bradyarrhythmias. European heart journal 10 (1989): 473-478.

9. Kuryshev YA, Brittenham GM, Fujioka H, et al. Decreased sodium and increased transient outward potassium currents in iron-loaded cardiac myocytes. Implications for the arrhythmogenesis of human siderotic heart disease. Circulation 100 (1999): 675-683.

10. Khode VH, Kammar KF. QTc changes in non-pregnant females with severe iron deficiency anaemia. 6 (2012): 777-779.

11. Detterich J, Noetzli L, Dorey F, et al. Electrocardiographic consequences of cardiac iron overload in thalassemia major. American journal of hematology 87 (2012): 139-144.

12. Scheller B, Pipa G, Kertscho H, et al. Low hemoglobin levels during normovolemia are associated with electrocardiographic changes in pigs. Shock 35 (2011): 375-381.

13. Mozos I, Serban C, Mihaescu R. Anemia and the QT interval in hypertensive patients. International Journal of Collaborative Research on Internal Medicine and Public Health 4 (2012): 2084.

14. Yamaguchi M, Shimizu M, Ino H, et al. T wave peak-to-end interval and QT dispersion in acquired long QT syndrome: a new index for arrhythmogenicity. Clinical science (London, England: 1979) 105 (2003): 671-676.

15. Chaudry II, Qamar S, Spodick DH. QT interval effects of normal QRS variation. American Journal of Cardiology 74 (1994): 628-629.

16. Sundqvist K, Sylven C. Cardiac repolarization properties during standardized exercise test as studied by QT, QT peak and terminated T-wave intervals. Clinical physiology (Oxford, England) 9 (1989): 419-425.

Citation: Huseyin Avni Findikli, Ayse Sahin Tutak. Evaluation of the Platelet Indices in Patients with Subclinical Hypothyroidism. Archives of Clinical and Biomedical Research 2 (2018): 233-238.

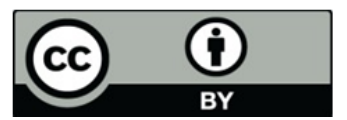

This article is an open access article distributed under the terms and conditions of the Creative Commons Attribution (CC-BY) license 4.0 